\title{
Hypertensive brainstem encephalopathy: a diagnosis often overlooked
}

\author{
Authors: Yin Yin $\operatorname{Tan}^{\mathrm{A}}$ and Kenny $\operatorname{Tan}^{\mathrm{B}}$
}

\begin{abstract}
Hypertensive encephalopathy (HE) is a subset of posterior reversible encephalopathy syndrome. It typically involves the posterior supratentorial structures, but variations do occur. However, isolated brainstem involvement in $\mathrm{HE}$ is rare, with a few cases reported in the literature. Herein, we report a case of acute hypertensive brainstem encephalopathy in which the patient had mild symptoms with very high blood pressure and normal neurological examination. The computed tomography of the brain showed diffuse hypodensity at brainstem. The patient's symptoms improved drastically after hypertension had been controlled. Marked clinical-radiologic dissociation in this particular case was highly suggestive of hypertensive brainstem encephalopathy. Prompt recognition of the condition and aggressive treatment of hypertension in such patients is crucial to relieve oedema and to prevent life-threatening progression. Nevertheless, there is still a lack of awareness among physicians and radiologists regarding this rare clinical entity.
\end{abstract}

KEYWORDS: Hypertensive brainstem encephalopathy, hypertension, computed tomography, clinical-radiological dissociation, reversible

\section{Case presentation}

A 52-year-old man presented to the emergency department with worsening occipital headache and giddiness over 2 days. He denied weakness, blurring of vision or altered sensation. In the emergency department, his blood pressure was $267 / 159 \mathrm{mmHg}$, pulse rate of 94 beats/min, and body temperature of $37.1^{\circ} \mathrm{C}$.

On further questioning, he was diagnosed with hypertension and chronic kidney disease 2 years ago. During the initial period of diagnosis, he was prescribed antihypertensive medication. However, he had defaulted follow-up and treatment for the past year.

Physical examination revealed full (15/15) Glasgow coma scale with no cerebellar signs. There was no evidence of focal

Authors: A physician, Langkawi Hospital, Langkawi, Malaysia; ${ }^{B}$ consultant neurologist, Loh Guan Lye Specialist Centre, Penang, Malaysia neurologic deficit, and his plantar reflexes were normal. Muscular strength was normal with 5/5 on the Medical Research Council strength scale for both bilateral upper limbs and lower limbs. The rest of the physical examinations were unremarkable. His blood investigations were normal except notable serum creatinine of $181 \mu \mathrm{mol} / \mathrm{L}$.

The patient was sent for an urgent computed tomography (CT) of the brain. The examination showed that the pons and midbrain were markedly and diffusely hypodense. There was no evidence of obstructive hydrocephalus. The parietal-occipital regions were relatively spared. The $\mathrm{CT}$ of the brain was reported by the radiologist as a massive brainstem infarct. Magnetic resonance imaging (MRI) examination was not done in view of limited resources in the hospital.

\section{Diagnosis}

Hypertensive brainstem encephalopathy was the most pertinent diagnosis in view of the marked clinical-radiological dissociation. The CT findings in our patient can represent diffuse glioma, infectious brainstem encephalitis, Wernicke's encephalopathy and central pontine myelinolysis. However, the relatively mild symptom and lack of cranial nerve finding with absence of focal neurological deficit despite extensive brainstem involvement in neuroimaging are highly suggestive of isolated brainstem involvement in hypertensive encephalopathy (HE). A normal blood sodium level made central pontine myelinolysis unlikely. There was no history to suggest Wernicke's encephalopathy as he denied alcohol consumption.

\section{Initial management and prognosis}

He was admitted to the general ward for blood pressure control. Continuous intravenous pump infusion of nitroglycerin was administered and he was commenced on oral antihypertensive medications.

\section{Case progression and outcome}

His symptoms resolved completely at day 2 of admission and his blood pressure was normalised after 5 days of hospitalisation. Follow-up CT of the brain after blood pressure normalisation showed almost complete reversal of the brainstem hypodensity. He was discharged with 3 types of oral antihypertensive medication. 
Fig 1. Computed tomography (CT) upon admission. a) Sequential axial CT of the upper brainstem show diffuse hypodensity of the pons (long black arrow). b) Hypodense areas are also visible at bilateral subthalamic nuclei (white arrows). c) The ambient cistern is preserved.
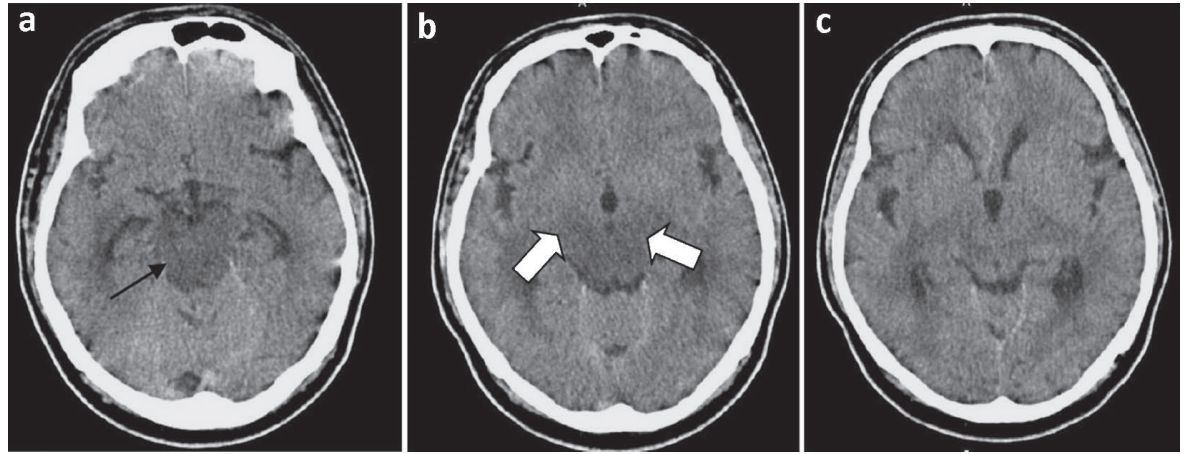

\section{Discussion}

$\mathrm{HE}$ is an acute syndrome of heterogeneous aetiologies characterised by acute hypertension associated with various neurological symptoms and characteristic neuroimaging findings. The common clinical signs and symptoms of the condition include encephalopathy, seizures, headache, visual disturbances, and focal neurological deficits. ${ }^{1} \mathrm{HE}$ is a relatively rare presentation but important to diagnose as the potential outcome ranges from the universally fatal to the completely reversible.

HE results from acute elevation of blood pressure beyond the upper limits of cerebral autoregulation. ${ }^{2}$ The neurologic syndrome in $\mathrm{HE}$ is now believed to be caused by vasogenic oedema secondary to failure of cerebral autoregulation. ${ }^{3}$ When systemic blood pressure rises over the autoregulatory threshold of the cerebral vasculature, it results in dilatation of cerebral arterioles and thus causes brain hyperperfusion. Subsequently, vasogenic oedema occurs as a result of breakdown of blood-brain barrier, with transudation of fluid and protein material. ${ }^{2,4}$

The similar clinical and radiological manifestations have been seen more commonly in patients with comorbid conditions, such as systemic lupus, cryoglobulinaemia, or haemolytic uraemic syndrome. ${ }^{5-7}$ Cyclosporine, cisplatin and tacrolimusrelated encephalopathy is reported to be associated with $\mathrm{HE}^{2,8,9}$
Notably, renal insufficiency is the risk factor in our case report as association between $\mathrm{HE}$ and renal insufficiency has been reported. $^{2}$

In view of wide differential diagnosis, neuroimaging plays an essential role in the diagnosis of HE. The findings are often apparent on CT, but best demonstrated on MRI. Diffusion weighted imaging (DWI) is helpful in HE cases because lack of restricted diffusion can rule out infarction, which is the most crucial differential diagnosis. Predominantly, it involves posterior supratentorial areas with typical parietal-occipital changes. Brainstem involvement is not infrequent but is commonly associated with the more typical supratentorial lesions which were absent in our patient. ${ }^{10}$

Marked clinical-radiological dissociation in this case rules out major brain stem infarction and tumour. Rapid clinical and radiological improvement after normalisation of the blood pressure alone exclude other possible differential diagnoses and confirm the diagnosis of hypertensive brainstem encephalopathy. Recognition of the brainstem variant of HE is paramount so that prompt treatment can be initiated to reverse posterior fossa oedema and hence prevent potential life-threatening complications. Radiologists may be the first to notice this alarming appearance on the brainstem, however interpretation of clinical symptoms and radiological findings, especially in the presence of 'clinical-radiological dissociation', should alert the clinicians and radiologists to suspect the diagnosis of hypertensive brainstem encephalopathy.
Fig 2. Follow-up computed tomography (CT). Sequential axial $\mathrm{CT}$ images at of the upper brainstem show almost complete resolution of the hypodense appearance of pons and subthalamic nuclei bilaterally.
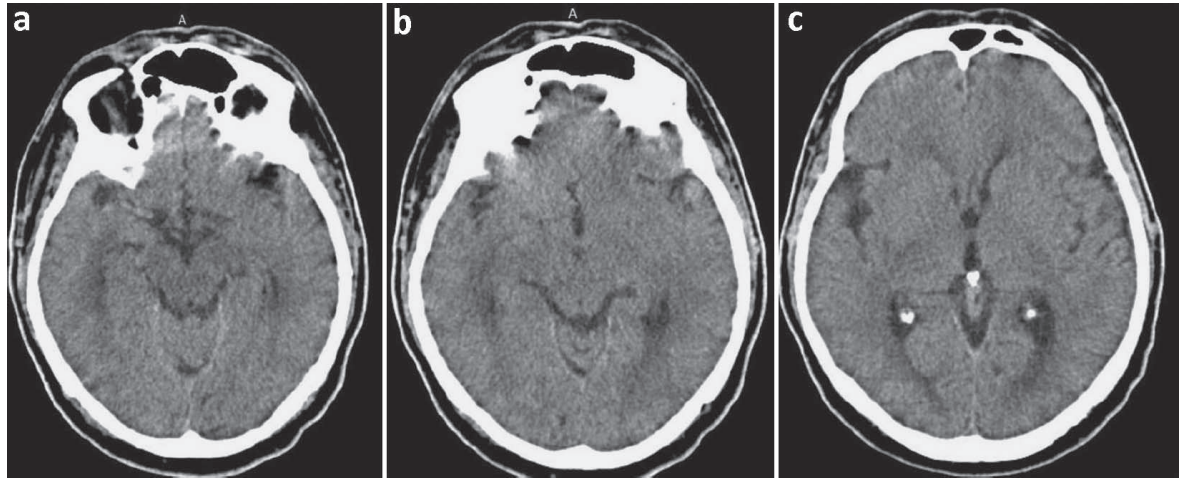


\section{Key learning points}

> Hypertensive brainstem encephalopathy is a rare entity and can be life threatening.

> Diagnosis of hypertensive brainstem encephalopathy should be considered in the context of high blood pressure, normal or minimal neurological findings with extensive brainstem involvement in neuroimaging (clinical-radiological dissociation).

$>$ Prompt and rapid initiation of treatment in suspected cases is important to prevent fatal outcome.

> Identification of predisposing risk factors can be useful in diagnosis of hypertensive encephalopathy.

> MRI is helpful for diagnostic purposes whenever available. However, treatment should not be delayed in the setting without MRI facility.

> Hypertensive brainstem encephalopathy is highly reversible by aggressive blood pressure control before progression to lifethreatening complications.

\section{References}

1 Li Y, Gor D, Walicki D et al. Spectrum and potential pathogenesis of reversible posterior leukoencephalopathy syndrome. J Stroke Cerebrovasc Dis 2012;21:873-82.

2 Hinchey J, Chaves C, Appignani B et al. A reversible posterior leukoencephalopathy syndrome. N Engl J Med 1996;334:494-500.

3 Schwartz RB, Jones KM, Kalina P et al. Hypertensive encephalopathy: findings on CT, MR imaging, and SPECT imaging in 14 cases. AJR Am J Roentgenol 1992;159:379-83.
4 Weingarten K, Barbut D, Filippi C, Zimmerman RD. Acute hypertensive encephalopathy: findings on spinecho and gradient-echo MR imaging. AJR Am J Roentgenol 1994;162:665-70.

5 Leroux G, Sellam J, Costedoat-Chalumeau N et al. Posterior reversible encephalopathy syndrome during systemic Lupus erythematosus: four new cases and review of the literature. Lupus 2008;17:139-47.

6 Hodson AK, Doughty RA, Norman ME. Acute encephalopathy, streptococcal infection, and cryoglobulinemia. Arch Neurol 1978;35:43-4.

7 Port JD, Beauchamp NJ Jr. Reversible intracerebral pathologic entities mediated by vascular autoregulatory dysfunction. Radiographics 1998;18:353-67.

8 Schwartz RB, Bravo SM, Klufas RA et al. Cyclosporine neurotoxicity and its relationship to hypertensive encephalopathy: $\mathrm{CT}$ and MR findings in 16 cases. AJR Am J Roentgenol 1995;165: 627-31.

9 Ito $Y$, Arahata $Y$, Goto $Y$ et al. Cisplatin neurotoxicity presenting as reversible posterior leukoencephalopathy syndrome. AJNR Am J Neuroradiol 1998;19:415-7.

10 Casey SO, Sampaio RC, Michel E, Truwit CL. Posterior reversible encephalopathy syndrome: utility of fluid-attenuated inversion recovery MR imaging in the detection of cortical and subcortical lesions. AJNR Am J Neuroradiol 2000;21:1199-206.

Address for correspondence: Dr Yin Yin Tan, Department of Internal Medicine, Langkawi Hospital, Bukit Tekuh, Jalan Padang Mat Sirat, 07000 Langkawi, Kedah, Malaysia. Email: tanyinyin2006@gmail.com

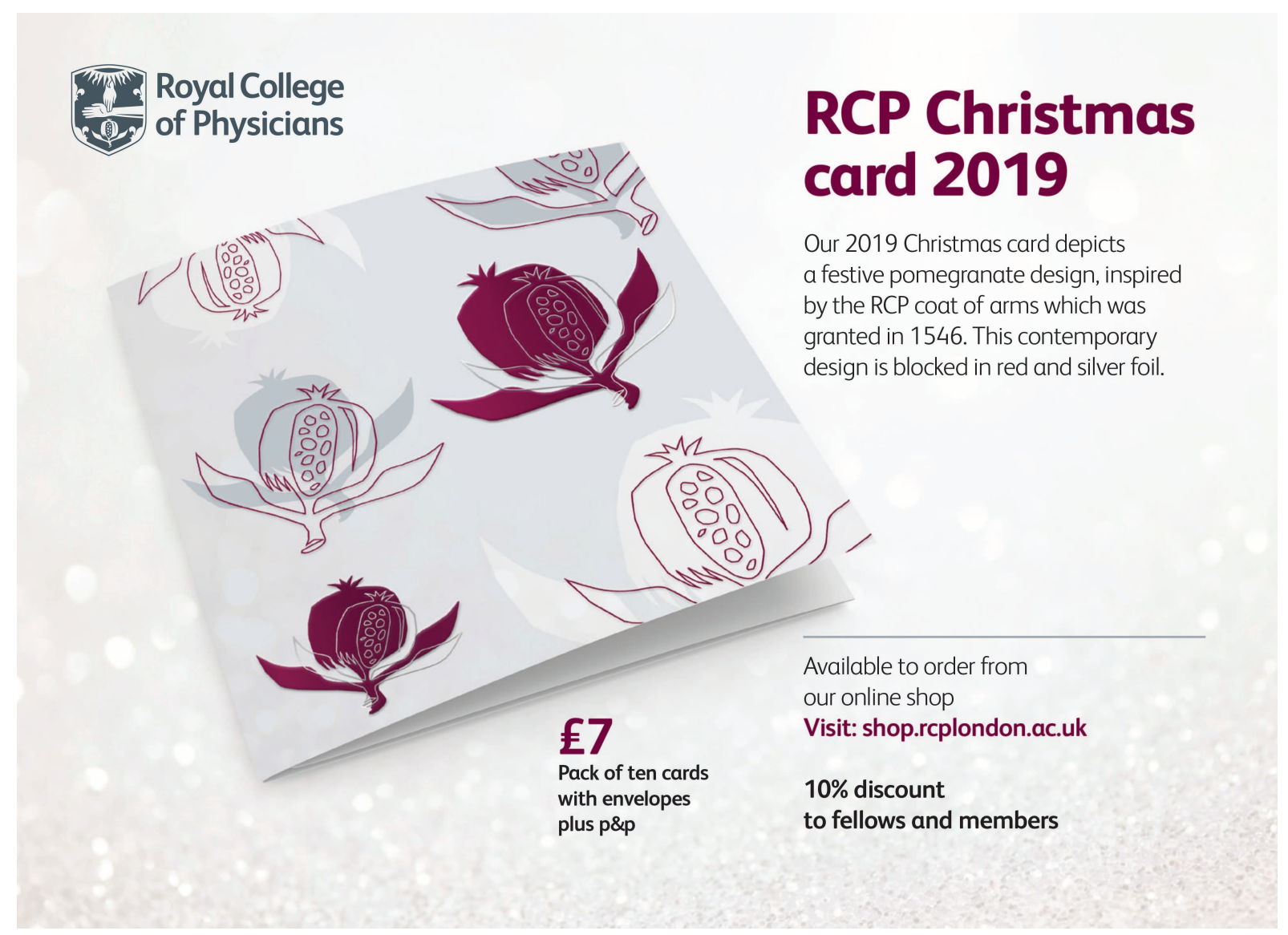

